\title{
Home Unattended Portable Monitoring and Automatic CPAP Titration in Patients with High Risk for Moderate to Severe Obstructive Sleep Apnea
}

\author{
Ersilia Tedeschi MD, Pierluigi Carratù MD PhD, Mario Francesco Damiani MD, \\ Valentina Anna Ventura MD, Riccardo Drigo MD, Edda Enzo MD, Alberto Ferraresso MD, \\ Geo Sasso MD, Franco Maria Zambotto MD, and Onofrio Resta MD
}

\begin{abstract}
BACKGROUND: Obstructive sleep apnea is a disorder characterized by recurrent obstruction of the upper airways during sleep. The high prevalence of this disease led to proposed new strategies based on the home evaluation and management of patients. OBJECTIVE: To compare home unattended portable monitoring and automatic CPAP titration with attended in-laboratory analysis, in a sample of patients with high risk for moderate to severe obstructive sleep apnea. METHODS: We enrolled 131 subjects, who were randomly divided into 2 groups: the home group $(n=66)$ was diagnosed and titrated at home; the laboratory group $(n=65)$ was analyzed in the sleep laboratory of our hospital. Diagnostic evaluations were carried out with portable monitoring at home, and with polysomnography in the sleep laboratory. Titration of CPAP was performed with the same automatic CPAP device in both groups. RESULTS: At the end of the study, 13 (19\%) subjects had dropped out of the home group, and $9(14 \%)$ of the laboratory group $(P=.50)$. There were no significant differences among groups in both baseline and with-CPAP values of apneahypopnea index, oxygen desaturation index, and total sleep time with $\mathrm{S}_{\mathrm{pO}_{2}}$ below $90 \%$. In the home group, the therapeutic pressure values reached at the end of each unattended home titration night were similar. CONCLUSIONS: A home diagnosis and titration approach should be considered in a subset of patients with obstructive sleep apnea. A single unattended titration night is sufficient to determine the therapeutic pressure. Key words: obstructive sleep apnea; OSA; CPAP; automatic CPAP titration; home titration; portable monitoring. [Respir Care 2013;58(7):1178-1183. () 2013 Daedalus Enterprises]
\end{abstract}

\section{Introduction}

Obstructive sleep apnea (OSA) is a disorder characterized by recurrent obstruction of the upper airway during

Drs Tedeschi, Carratù, Damiani, Ventura, and Resta are affiliated with the Institute of Respiratory Disease, University of Bari, Bari, Italy. Drs Drigo, Enzo, Ferraresso, Sasso, and Zambotto are affiliated with the Center of Sleep Breathing Medicine, Unità Operativa Complessa de Pneumologie, Hospital Santa Maria del Prato, Feltre, Belluno, Italy.

The authors have disclosed no conflicts of interest.

Correspondence: Pierluigi Carratù MD PhD, Institute of Respiratory Disease, University of Bari, Piazza Giulio Cesare 11, Bari, Italy 70124. E-mail: pierluigicarratu@yahoo.com.

DOI: $10.4187 /$ respcare.01939 sleep, resulting in intermittent oxygen desaturation, daytime sleepiness, and decreased quality of life. The standard approach to diagnosis and titration of patients with suspected OSA consists of polysomnography (PSG), followed by manual titration of CPAP during attended PSG. ${ }^{1,2}$ OSA is a highly prevalent disease: in 1993 , Young et al $^{3}$ reported that moderate to severe OSA affects $4 \%$ of adult women and $9 \%$ of adult men, and a large proportion of these people are undiagnosed. These results likely underestimate the current prevalence of OSA due to an important increase in obesity over the past 2 decades. ${ }^{4}$ The prevalence of this disease and its association with the onset of hypertension, ${ }^{5,6}$ cardiovascular diseases, ${ }^{7}$ stroke, ${ }^{8,9}$ increased motor vehicle accidents, ${ }^{10}$ and mortality ${ }^{9}$ have made it a major public health issue. On the other hand, patient wait time for in-laboratory attended PSG in many public 
healthcare systems is often too long; indeed, it can exceed 6 months. ${ }^{11}$ Moreover, in-laboratory PSG is expensive and time consuming. ${ }^{12}$ All these reasons led to proposal of new strategies based on the home evaluation and management of patients. ${ }^{13}$ Indeed, home portable monitoring is a cheap and easily accessible test. ${ }^{12}$ With regard to titration, there is evidence that certain automatic CPAP devices may be used at home to determine the therapeutic pressure. ${ }^{2}$ However, some doubts remain about this practice; in fact, the available evidence still cannot support a guideline recommendation. ${ }^{2}$ The aim of this study was to compare home unattended portable monitoring and automatic CPAP titration to an attended in-laboratory program, in a sample of patients with high risk for moderate to severe OSA.

\section{Methods}

\section{Subjects}

Between June 2008 and September 2009 we enrolled 131 subjects who were referred for their first sleep study for suspicion of OSA to our sleep center in the pulmonology section at Hospital Santa Maria del Prato, Feltre, Belluno, Italy. The inclusion criteria were:

- High suspicion of OSA, based on excessive daytime sleepiness (Epworth Sleepiness Scale score $\geq 10$ ), history of habitual snoring, witnessed apneas, and oxygen desaturation index $>30$, assessed during home night oximetry (Pulsox3i, Minolta, Tokyo, Japan)

- Absence of cardiovascular, respiratory, and neuromuscular comorbidities

- Subject lived within $100 \mathrm{~km}$ of the hospital

\section{Design of the Study}

This work was a randomized, prospective study, with 2 parallel arms. The demographic characteristics and Epworth Sleepiness Scale scores are shown in Table 1, with no differences between groups. Subjects were randomly divided into 2 groups: the home group $(n=66)$ was diagnosed and titrated at home; the laboratory group $(n=65)$ was evaluated in the sleep laboratory of our hospital. The randomization assignments were computer generated, using a block permutation method to select 1 of the 2 diagnostic and titration alternatives. Randomization was performed by the staff of our hospital, who had no direct participation in the study. Subjects in the home group were examined at home with a portable monitoring device. To assess the reliability of portable monitoring we evaluated the quality of the 3 main signals: oxygen saturation, thoracic/abdominal movements, and nasal air flow. If the subject required CPAP (apnea-hypopnea index [AHI]

\section{QUICK LOOK}

\section{Current knowledge}

Obstructive sleep apnea is characterized by recurrent obstruction of the upper airway during sleep, resulting in intermittent hypoxemia, daytime sleepiness, and decreased quality of life. The standard diagnosis of suspected obstructive sleep apnea consists of attended polysomnography, followed by manual PEEP titration.

\section{What this paper contributes to our knowledge}

In selected patients, home unattended sleep monitoring and automatic PEEP titration resulted in similar PEEP level, apnea-hypopnea index, oxygen desaturation index, and hypoxemia. A single unattended PEEP titration is sufficient to determine the therapeutic PEEP.

$\geq 5$ events/h) according to the international guidelines, ${ }^{14}$ he or she underwent unattended home automatic CPAP titration during 4 consecutive nights. To confirm the therapeutic pressure reached during automatic CPAP titration, the subject then underwent attended PSG with CPAP in the sleep laboratory. In all subjects we applied a pressure that represented the mean value from the pressures determined at the end of each night. The laboratory group was diagnosed with overnight PSG in the sleep laboratory. If the circumstances mentioned above for the home group were present, the laboratory group subject was titrated with automatic CPAP during one night in the laboratory. In the home group the therapeutic pressure was tested during PSG with CPAP in the laboratory. In both groups, all the diagnostic and therapeutic evaluations were performed during consecutive nights.

\section{Methods}

PSG was performed in the laboratory, using a recording device (Grass-Telefactor, Astro-Med, West Warwick, Rhode Island). The following signals were measured: nasal air flow (nasal cannula and thermocouple), thoracic and abdominal movements, arterial oxygen saturation, electrocardiogram, body position, and snoring.

The portable monitor used at home (Somtè PSG, Compumedics, Abbotsford, Victoria, Australia) recorded the following signals: nasal air flow, thoracic and abdominal movements, arterial oxygen saturation, electrocardiogram, body position, and snoring.

In the attended laboratory studies, a clinical laboratory technician provided continuous monitoring of parameters to identify and correct any sensor loss. Portable monitoring and PSG recordings were interpreted by 2 sleep med- 
Table 1. Demographic Characteristics of Subjects

\begin{tabular}{|c|c|c|c|c|}
\hline & $\begin{array}{l}\text { All Subjects } \\
\quad n=131\end{array}$ & $\begin{array}{c}\text { Home Group } \\
n=66\end{array}$ & $\begin{array}{l}\text { Laboratory } \\
\text { Group } \\
n=65\end{array}$ & $P$ \\
\hline Age, mean \pm SD y & $60.84 \pm 9.72$ & $59.5 \pm 9.18$ & $62.2 \pm 10.12$ & .11 \\
\hline Male, no. (\%) & $102(77)$ & $51(77)$ & $51(78)$ & .96 \\
\hline Female, no. $(\%)$ & $29(22)$ & $15(22)$ & $14(21)$ & .96 \\
\hline Body mass index, mean $\pm \mathrm{SD} \mathrm{kg} / \mathrm{m}^{2}$ & $34.17 \pm 5.9$ & $33.74 \pm 5.09$ & $34.61 \pm 6.64$ & .40 \\
\hline Neck circumference, mean \pm SD cm & $43.71 \pm 4.25$ & $43.15 \pm 3.52$ & $44.29 \pm 4.85$ & .12 \\
\hline Epworth Sleepiness Scale score, mean \pm SD & $12.74 \pm 2.62$ & $12.46 \pm 2.46$ & $13.03 \pm 2.75$ & .21 \\
\hline
\end{tabular}

icine physicians, according to the Associazione Italiana Pneumologi Ospedalieri guidelines. ${ }^{15}$

An AHI $\geq 5$ was necessary to diagnose OSA. ${ }^{14}$ Neither the PSG nor the portable monitoring were considered reliable if the quality of one of the main signals (oxygen saturation, thoracic/abdominal movements, nasal air flow) was poor for more than $20 \%$ of the recording time.

The home and laboratory titration of CPAP were performed with the same automatic CPAP device (RemStar Auto, Respironics, Murrysville, Pennsylvania). Each machine was used with the default settings, with the lower and upper pressure bounds set to 4 and $16 \mathrm{~cm} \mathrm{H}_{2} \mathrm{O}$, respectively. According to the manufacturer's instructions, the reference pressure level was the 90th percentile of the pressure determined by the device. Information recorded by the automatic CPAP device was examined by a sleep medicine physician. The recording was considered acceptable if the recording duration was at least 3 hours, and leaks were not present for more than $20 \%$ of the recording time, according to the manufacturer's instructions. In the home group the therapeutic pressure was considered reliable if we had at least 2 days of recording. Before titration, subjects in both groups received a demonstration, from a nurse, of the procedures to operate the machine and install the mask, and 20 min acclimatization session of automatic CPAP, during an afternoon session.

The study was approved by the institutional review board of Bari University General Hospital, and carried out in accordance with the principles of the Helsinki Declaration. All subjects gave prior written informed consent to take part in the study.

\section{Statistical Analysis}

Data are presented as mean \pm SD unless otherwise indicated. Differences between groups were analyzed with the Student $t$ test for independent samples. To compare the therapeutic pressure values determined during the 4-night home titration, analysis of variance was performed. A value of $P<.05$ was considered statistically significant. The analyses were made using statistics software (Statistica 6.1, StatSoft, Tulsa, Oklahoma).

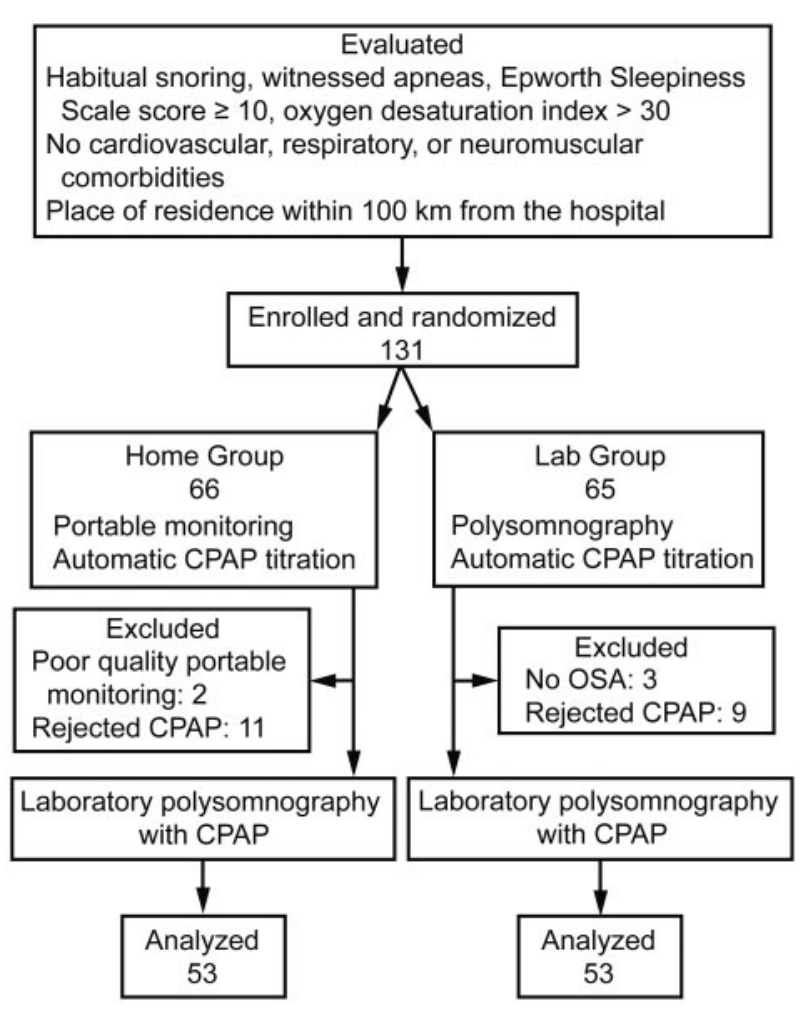

Figure. Study flow chart.

\section{Results}

The Figure shows the study flow chart. We enrolled 131 subjects. In the home group, 2 subjects' data were excluded because of poor quality portable monitoring. In addition, 4 subjects did not agree to undergo unattended automatic CPAP titration: 2 because of incapacity to wear the mask (claustrophobia), and 2 due to non-acceptance of long-term treatment. Seven did not complete the home titration of CPAP, due to: the automatic CPAP recording was not considered acceptable, due to excessive leakage (3 subjects); complaint of nasal stuffiness (2 subjects); mask discomfort (2 subjects).

In the laboratory group the quality of baseline PSG was good in all cases, but 3 subjects were excluded: 2 because 


\section{Home Unattended Portable Monitoring and Automatic CPAP Titration}

Table 2. Sleep Parameters at Baseline and With CPAP

\begin{tabular}{|c|c|c|c|c|}
\hline & $\begin{array}{l}\text { All Subjects } \\
n=106\end{array}$ & $\begin{array}{l}\text { Home Group } \\
n=53\end{array}$ & $\begin{array}{l}\text { Laboratory } \\
\text { Group } \\
n=53\end{array}$ & $P$ \\
\hline \multicolumn{5}{|c|}{$\begin{array}{l}\text { Apnea-hypopnea } \\
\text { index }\end{array}$} \\
\hline Baseline & $50.99 \pm 17.17$ & $48.77 \pm 15.79$ & $53.2 \pm 18.33$ & .18 \\
\hline With CPAP & $2.52 \pm 1.86$ & $2.39 \pm 1.99$ & $2.66 \pm 1.73$ & .45 \\
\hline \multicolumn{5}{|c|}{$\begin{array}{l}\text { Oxygen desaturation } \\
\text { index }\end{array}$} \\
\hline Baseline & $43.5 \pm 9.85$ & $42.79 \pm 9.29$ & $44.2 \pm 10.42$ & .46 \\
\hline With CPAP & $3.43 \pm 2.55$ & $3.22 \pm 2.12$ & $3.64 \pm 2.92$ & .39 \\
\hline \multicolumn{5}{|c|}{$\begin{array}{l}\text { Total sleep time with } \\
\qquad \mathrm{S}_{\mathrm{pO}_{2}}<90 \%\end{array}$} \\
\hline Baseline & $44.83 \pm 19.97$ & $43.6 \pm 18.97$ & $46.07 \pm 21.04$ & .52 \\
\hline With CPAP & $3.95 \pm 3.18$ & $3.64 \pm 3.14$ & $4.26 \pm 3.22$ & .31 \\
\hline
\end{tabular}

they were diagnosed with central sleep apnea, and 1 was found not to need CPAP (AHI $<5$ events/h). All the subjects underwent attended automatic CPAP titration, but 9 did not complete the laboratory titration, due to failure to fall asleep (3 subjects), dry mouth (3 subjects), nasal stuffiness ( 2 subjects), and mask discomfort (1 subject). The percentage of reliable baseline portable monitoring and PSG was similar between groups: 97\% (64/66) in the home group versus $100 \%(65 / 65)$ in the laboratory group $(P=.48)$. In the home group, $17 \%$ (11/64) of subjects refused CPAP before or during titration, with similar results in the laboratory group $(14 \%, 9 / 62, P=.86)$. At the end of the study, 13 (19\%) subjects had dropped out of the home group, and 9 (14\%) of the laboratory group, with no significant differences between groups $(P=.50)$. The remaining 106 completed the program. The therapeutic pressure values determined by automatic CPAP titration were not different between the groups: $13.3 \pm 1.7 \mathrm{~cm} \mathrm{H}_{2} \mathrm{O}$ was the mean value of the pressures determined at the end of 4 unmonitored titration nights, and $13.5 \pm 1.3 \mathrm{~cm} \mathrm{H}_{2} \mathrm{O}$ was the mean value of the pressures determined after a single titration night in the laboratory $(P=.49)$.

The therapeutic pressure values determined at the end of each unattended home titration night had no statistical differences between the nights: $13.2 \pm 1.7 \mathrm{~cm} \mathrm{H}_{2} \mathrm{O}$, $13.4 \pm 1.7 \mathrm{~cm} \mathrm{H} \mathrm{H}_{2} \mathrm{O}, 13.1 \pm 1.6 \mathrm{~cm} \mathrm{H}_{2} \mathrm{O}$, and $13.5 \pm 1.7 \mathrm{~cm} \mathrm{H}_{2} \mathrm{O}, P=.59$. This means that the pressures determined during any of the 4 unattended titration nights were similar to those determined during the one attended in-laboratory titration night. Table 2 shows the baseline and with-CPAP values of AHI, oxygen desaturation index, and total sleep time with $\mathrm{S}_{\mathrm{pO}_{2}}<90 \%$. There were no significant differences between the groups.

The number of subjects with severe OSA (AHI $>30$ events/h) was 50 in the home group and 51 in the laboratory group $(P>.99)$. The AHI normalized $(<5$ events $/ \mathrm{h})$ in the home group in all but 3 subjects, and in the laboratory group in all but $2(P>.99)$. The baseline sleep characteristics of subjects who did not undergo/complete automatic CPAP titration were similar to those who reached the end of the study (data not shown).

\section{Discussion}

This study demonstrated that for individuals with high risk of OSA, the home unattended diagnostic/therapeutic program is as effective as the hospital attended evaluation. With regard to the diagnosis of OSA, although the PSG is the gold standard, ${ }^{1}$ over the last decade it has widely been demonstrated that home portable monitoring is a valid and reliable test. ${ }^{16,17}$ Furthermore, recently, the Portable Monitoring Task Force of the American Academy of Sleep Medicine recommended that portable monitoring should be limited to subjects with a high risk for moderate to severe OSA, ${ }^{18}$ such as in our work. In agreement with the literature, ${ }^{19} 3 \%$ of the home portable monitoring was not reliable, with no significant differences between groups. As far as the titration is concerned, home unattended automatic CPAP represents a useful and accurate way to identify conventional CPAP setting outside the hospital.

Sériès is one of the pioneers of the home-based protocol. In his work, ${ }^{20}$ dating back to 2000 , a 1 - or 2 -week home automatic CPAP trial was done in 40 subjects, by establishing the reference pressure to be set on a fixed CPAP machine for 2 additional weeks, plus a control PSG with fixed CPAP. A significant improvement was demonstrated in sleep architecture and AHI, with normalization in almost all subjects, encouraging subsequent research. Later, other studies comparing in-laboratory manual titration to unattended automatic CPAP showed similar results between groups in AHI, sleepiness score, and quality of life. ${ }^{21,22}$ Our work, on one hand, confirmed these findings: we found a significant improvement in AHI, oxygen desaturation index, and total sleep time with $\mathrm{S}_{\mathrm{pO}_{2}}<90 \%$ in the group titrated at home, without significant differences between the groups. On the other hand, our study is different from the studies mentioned above in 2 important aspects: first, the method of titration. In fact, in the Sériès work, ${ }^{20}$ all subjects underwent unmonitored titration with automatic CPAP, and there was no laboratory group. The other 2 studies 21,22 included attended groups, and the subjects underwent manual titration during PSG. We compared 2 groups, attended versus unattended, both titrated with automatic CPAP. Indeed, our goal was not to evaluate the effectiveness of the device, but rather to test its usefulness outside the hospital. Thus, we thought that the presence of manual titration in the laboratory group could be a confounding factor for analysis. Second, this protocol did not include follow-up of the subjects, because we did 


\section{Home Unattended Portable Monitoring and Automatic CPAP Titration}

not intend to assess patient adherence or adaptation to CPAP.

The required duration of home automatic CPAP monitoring still remains to be determined. Bosi and Fiorentino ${ }^{23}$ reported that patients experienced good quality sleep after about 10 days of home titration. Bachour et al, ${ }^{24}$ who evaluated cost-effectiveness, found that the optimal trial duration is 5 days. In a large multicenter study, Masa et $\mathrm{al}^{21}$ showed the achievement of automatic titration at home after 1-3 nights. Our protocol consisted of 4-day unmonitored automatic CPAP titration. Similar to other works, ${ }^{24,25}$ between home titration nights we found no significant differences in the therapeutic pressure values. This result led us to consider the last 3 nights unnecessary. However, it is important to underline that not all subjects achieved normalized AHI. In this regard we would like to highlight the important role of the attended in-laboratory PSG with fixed CPAP, performed after a 4-night home titration, to confirm the therapeutic pressure values. In 2007 the American Academy of Sleep Medicine declared that a home-based protocol could be used without any further confirmation. ${ }^{18}$ Nevertheless, there is evidence that, by repeating manual titration for more nights in the laboratory, we can obtain different values, by 1,2 , and sometimes $3 \mathrm{~cm} \mathrm{H}_{2} \mathrm{O}^{26}$ Moreover, recently Mulgrew et al ${ }^{27}$ found an AHI of $>10$ events/h in $25 \%$ of manually titrated subjects, when examined over the time. Both these results and our work suggest that an attended confirming PSG could be a valid method to correct pressure values when it is needed.

The reliability of the home-based protocol is also demonstrated by the absence of significant differences between the groups in the percentage of subjects who dropped out of the study. Indeed, the numbers of both poor quality recordings and early refusal of CPAP were similar between the 2 groups. The literature has reported a wide variability in percentage of patients who refuse CPAP before/during home titration..$^{24,25,28}$ In our work, $17 \%$ refused CPAP in the home group, versus $14 \%$ in the laboratory group.

An important limitation of this study is that we enrolled subjects with high risk for moderate to severe OSA. Thus, our results apply only to this patient population. On the other hand, we followed the recommendations, mentioned above, provided by the American Academy of Sleep Medicine. ${ }^{18}$ Additional studies are required to measure the effects of home automatic CPAP titration on outcomes such as sleepiness and quality of life.

\section{Conclusions}

In conclusion, the home diagnosis and titration approach should be considered in a subset of patients with OSA. A single unattended titration night is sufficient to determine the therapeutic pressure. In subjects titrated both in the home and the laboratory setting, a confirming PSG may be useful.

\section{REFERENCES}

1. Douglas NJ, Thomas S, Jan MA. Clinical value of polysomnography. Lancet 1992;339(8789):347-350.

2. Morgenthaler TI, Aurora RN, Brown T, Zak R, Alessi C, Boehlecke $\mathrm{B}$, et al. Practice parameters for the use of autotitrating continuous positive airway pressure devices for titrating pressures and treating adult patients with obstructive sleep apnea syndrome: an update for 2007. An American Academy of Sleep Medicine report. Sleep 2008; 31(1):141-147.

3. Young T, Palta M, Dempsey J, Skatrud J, Weber S, Badr S. The occurrence of sleep-disordered breathing among middle-aged adults. N Engl J Med 1993;328(17):1230-1235.

4. Young T, Peppard PE, Taheri S. Excess weight and sleep-disordered breathing. J Appl Physiol 2005;99(4):1592-1599.

5. Kales A, Bixler EO, Cadieux RJ, Schneck DW, Shaw LC 3rd, Locke TW, et al. Sleep apnoea in a hypertensive population. Lancet 1984; 2(8410):1005-1008.

6. Peppard PE, Young T, Palta M, Skatrud J. Prospective study of the association between sleep-disordered breathing and hypertension. N Engl J Med 2000;342(19):1378-1384.

7. Malone S, Liu PP, Holloway R, Rutherford R, Xie A, Bradley TD. Obstructive sleep apnoea in patients with dilated cardiomyopathy: effects of continuous positive airway pressure. Lancet 1991; 338(8781):1480-1484.

8. Partinen M, Palomäki H. Snoring and cerebral infarction. Lancet 1985;2(8468):1325-1326.

9. Yaggi HK, Concato J, Kernan WN, Lichtman JH, Brass LM, Mohsenin V. Obstructive sleep apnea as a risk factor for stroke and death. N Engl J Med 2005;353(19):2034-2041.

10. George CF. Reduction in motor vehicle collisions following treatment of sleep apnoea with nasal CPAP. Thorax 2001;56(7):508-512.

11. Flemons WW, Douglas NJ, Kuna ST, Rodenstein DO, Wheatley J. Access to diagnosis and treatment of patients with suspected sleep apnea. Am J Respir Crit Care Med 2004;169(6):668-672.

12. Parra O, García-Esclasans N, Montserrat JM, García Eroles L, Ruíz J, López JA, et al. Should patients with sleep apnoea/hypopnoea syndrome be diagnosed and managed on the basis of home sleep studies? Eur Respir J 1997;10(8):1720-1724.

13. Mulgrew AT, Fox N, Ayas NT, Ryan CF. Diagnosis and initial management of obstructive sleep apnea without polysomnography: a randomized validation study. Ann Intern Med 2007;146(3):157-166.

14. Epstein LJ, Kristo D, Strollo PJ Jr, Friedman N, Malhotra A, Patil SP, et al; on behalf of Adult Obstructive Sleep Apnea Task Force of the American Academy of Sleep Medicine. Clinical guideline for the evaluation, management and long-term care of obstructive sleep apnea in adults. J Clin Sleep Med 2009;5(3):263-276.

15. Gruppo di studio sui disturbi respiratori nel sonno dell'Associazione Italiana Pneumologi Ospedalieri. Linee guida per la refertazione della polisonnografia e dei monitoraggi cardiorespiratori per sospetta sindrome della apnee ostruttive nel sonno in soggetti adulti. Rassegna di Patologia dell'Apparato Respiratorio 2003;18:305-308. Article in Italian.

16. Chiner E, Signes-Costa J, Arriero JM, Marco J, Fuentes I, Sergado A. Nocturnal oximetry for the diagnosis of the sleep apnoea hypopnoea syndrome: a method to reduce the number of polysomnographies? Thorax 1999;54(11):968-971.

17. Lloberes P, Sampol G, Levy G, Aristizabal D, Sagales T, De la Calzada $\mathrm{M}$, et al. Influence of setting on unattended respiratory 


\section{Home Unattended Portable Monitoring and Automatic CPAP Titration}

monitoring in the sleep apnoea/hypopnoea syndrome. Eur Respir J 2001;18(3):530-534.

18. Collop NA, Anderson WM, Boehlecke B, Claman D, Goldberg R, Gottlieb DJ, et al; on behalf of Portable Monitoring Task Force of the American Academy of Sleep Medicine. Clinical guidelines for the use of unattended portable monitors in the diagnosis of obstructive sleep apnea in adult patients. J Clin Sleep Med 2007;3(7):737-747.

19. Flemons WW, Littner MR, Rowley JA, Gay P, Anderson WM, Hudgel DW, et al. Home diagnosis of sleep apnea: a systematic review of the literature. An evidence review cosponsored by the American Academy of Sleep Medicine, the American College of Chest Physicians, and the American Thoracic Society. Chest 2003; 124(4):1543-1579.

20. Sériès F. Accuracy of an unattended home CPAP titration in the treatment of obstructive sleep apnea. Am J Respir Crit Care Med 2000;162(1):94-97.

21. Masa JF, Jiménez A, Durán J, Capote F, Monasterio C, Mayos M, et al. Alternative methods of titrating continuous positive airway pressure: a large multicenter study. Am J Respir Crit Care Med 2004; 170(11):1218-1224.

22. Skomro RP, Gjevre J, Reid J, McNab B, Ghosh S, Stiles M, et al. Outcomes of home-based diagnosis and treatment of obstructive sleep apnea. Chest 2010;138(2):257-263.
23. Bosi M, Fiorentino F. Validità della titolazione con autoCPAP e con monitoraggio cardiorespiratorio per il trattamento della sindrome delle apnee ostruttive nel sonno. Rassegna di Patologia dell' Apparato Respiratorio 2007;22:381-390. Article in Italian.

24. Bachour A, Virkkala JT, Maasilta PK. AutoCPAP initiation at home: optimal trial duration and cost-effectiveness. Sleep Med 2007;8(78):704-710

25. Sériès F, Plante J, Lacasse Y. Reliability of home CPAP titration with different automatic CPAP devices. Respir Res 2008;9:56.

26. Wiest GH, Fuchs FS, Harsch IA, Pour Schahin S, Lampert S, Brueckl $\mathrm{WM}$, et al. Reproducibility of a standardized titration procedure for the initiation of continuous positive airway pressure therapy in patients with obstructive sleep apnoea. Respiration 2001;68(2):145150

27. Mulgrew AT, Lawati NA, Ayas NT, Fox N, Hamilton P, Cortes L, Ryan CF. Residual sleep apnea on polysomnography after 3 months of CPAP therapy: clinical implications, predictors and patterns. Sleep Med 2010;11(2):119-125.

28. Cross MD, Vennelle M, Engleman HM, White S, Mackay TW, Twaddle S, Douglas NJ. Comparison of CPAP titration at home or the sleep laboratory in the sleep apnea hypopnea syndrome. Sleep 2006;29(11):1451-1455. 